\title{
Advanced Characterization of Interfaces and Thin Films
}

\author{
RITESH SACHAN (i) ${ }^{1,3,4}$ and VIKAS TOMAR ${ }^{2,5}$ \\ 1.-Materials Science and Technology Division, Oak Ridge National Laboratory, Oak Ridge, \\ TN 37831, USA. 2.-School of Aeronautics and Astronautics, Purdue University, West Lafayette, \\ IN 47906, USA. 3.—e-mail: sachan.ritesh@gmail.com. 4.—e-mail: sachanr@ornl.gov. 5.—e-mail: \\ Tomar@purdue.edu
}

One of the most critical issues in materials science is a control of materials properties through a control of local material features. A major emphasis has been placed on tuning material properties by a modification of underlying chemistry of surfaces and interfaces. In thin films and coatings, the effect of surfaces and interfaces is significant as a result of a major interplay of strain and atomic disordering at high surface-to-material volume ratio values. Advanced characterization methods can play an important role in developing a fundamental understanding of underlying mechanisms that affect surface and interface mechanical and functional properties.

This topic covers research work on advanced characterization of materials interfaces at multiple length scales in metal, alloys, ceramics, and polymers by various in situ and ex situ experimental techniques such as x-ray diffraction (XRD), scanning electron microscopy (SEM), transmission electron microscopy (TEM), atomic force microscopy (AFM), surface-enhanced Raman spectroscopy (SERS), and neutron diffraction. The topic also involves research work focusing on developing an understanding of materials interface behavior by theoretical calculation and modeling approaches (e.g., finite element analysis, density functional theory, molecular dynamics, and Monte Carlo simulations).

In this commentary, we highlight experimental and theoretical advancements in various research works published in this issue. S. Awasthi et al. report insights on the tribology studies on nickel-diamond coatings at multiple length scales by implementing several experimental characterization schemes such as, e.g., XRD, SEM, and SERS. In another work, R.P. Chaudhary et al. present a quantitative study focusing on the adsorption of azo-dye molecules on

Ritesh Sachan and Vikas Tomar are the JOM advisors for the Thin Films \& Interfaces Committee of the TMS Functional Materials Division, and guest editors for the topic Advanced Characterization of Interfaces and Thin Films in this issue. graphene oxide thin films using the optical spectroscopy. S. Abedrabbo et al. provide the results of the enhancement of the silicon band-edge emission at $\mathrm{Si} / \mathrm{ErO}_{2}$ interfaces using the photoluminescence spectroscopy technique. R. Yu et al. show a novel way to measure the localized temperature in the planar stacks of solid oxide fuel cell modules. A combination of experiments and molecular dynamics simulations is used by $\mathrm{H}$. Li et al. to understand the hydration and solvation behavior of layered structured montmorillonite during the delamination process. M. Reza Nasresfahani et al. carried out investigations to evaluate the application of the theoretical LiptonGlicksman-Kurz model to predict the dendritic growth during the solidification process by applying an external electric field.

Overall, this topic on advanced characterization of interfaces and thin films discusses the research works implementing a broad spectrum of advanced characterization methods with emphasis on interface chemistry and its relation to material performance.

The following articles being published under the topic of "Advanced Characterization of Interfaces and Thin Films" provide excellent details and research on the subject. To download any of the articles, follow the url http://link.springer.com/jour nal/11837/69/2/page/ 1 to the table of contents page for the February 2017 issue (vol. 69, no. 2).

- "Multi-Length Scale Tribology of Electrophoretically Deposited Nickel-Diamond Coating" by Shikha Awasthi, Sneha Goel, Chandra Prabha Pandey, and Kantesh Balani.

- "Quantification of Adsorption of Azo dye Molecules on Graphene Oxide using Optical Spectroscopy" by Raghvendra Pratap Chaudhary, Pranav Bhagwan Pawar, Kumar Vaibhav, Sumit Saxena, and Shobha Shukla.

- "Evidence of Silicon Band-Edge Emission Enhancement When Interfaced with $\mathrm{SiO}_{2}: \mathrm{Er}$ Films" by S. Abedrabbo, A.T. Fiory, and N.M. Ravindra. 
- "Probing Temperature Inside Planar SOFC Short Stack, Modules, and Stack Series" by Rong Yu, Wanbing Guan, and Xiao-Dong Zhou.

- "Comparison Study on the Effect of Interlayer Hydration and Solvation on Montmorillonite Delamination" by Hongliang Li, Shaoxian Song,
Yunliang Zhao, Yuri Nahmad, and Tianxing Chen.

- "Evaluation of Lipton-Glicksman-Kurz Model for Free Dendritic Growth Under an Applied Electric Field” by Mohamad Reza Nasresfahani, Behzad Niroumand, and Ahmad Kermanpur. 